\title{
The cool magnetic DAZ white dwarf NLTT 10480^
}

\begin{abstract}
A. Kawka and S. Vennes
Astronomický ústav, Akademie věd České republiky, Fričova 298, 25165 Ondřejov, Czech Republic

e-mail: [kawka; vennes]@sunstel.asu.cas.cz

Received 14 April 2011 / Accepted 1 June 2011

ABSTRACT

We have identified a new cool magnetic white dwarf in the New Luyten Two-Tenths (NLTT) catalogue. The high proper-motion star NLTT $10480\left(\mu=0.5^{\prime \prime} \mathrm{yr}^{-1}\right)$ shows weak Zeeman-split lines of calcium as well as characteristic $\mathrm{H} \alpha$ and $\beta$ Zeeman triplets. Using VLT X-shooter spectra, we measured a surface-averaged magnetic field $B_{\mathrm{S}} \sim 0.5 \mathrm{MG}$. The relative intensity of the $\pi$ and $\sigma$ components of the calcium and hydrogen lines imply a high inclination $\left(i \gtrsim 60^{\circ}\right)$. The optical-to-infrared $V-J$ colour index and the $\mathrm{Ca} \mathrm{I} / \mathrm{Ca}$ II ionization balance indicate a temperature between 4900 and $5200 \mathrm{~K}$, while the Balmer line profiles favour a higher temperature of $5400 \mathrm{~K}$. The discrepancy is potentially resolved by increasing the metallicity to $0.03 \times$ solar, hence increasing the electron pressure. However, the measured calcium abundance and abundance upper limits for other elements $(\mathrm{Na}, \mathrm{Al}, \mathrm{Si}$, and $\mathrm{Fe})$ imply a low photospheric metallicity $\lesssim 10^{-4}$ solar. Assuming diffusion steady-state, a calcium accretion rate of $\log \dot{M}\left(\mathrm{~g} \mathrm{~s}^{-1}\right)=5.6 \pm 0.3$ is required to sustain a calcium abundance of $\log n(\mathrm{Ca}) / n(\mathrm{H})=-10.30 \pm 0.30$ in the white dwarf atmosphere. We examine the implications of this discovery for the incidence of planetary debris and weak magnetic fields in cool white dwarf stars.
\end{abstract}

Key words. white dwarfs - stars: individual: NLTT 10480 - stars: atmospheres - stars: abundances

\section{Introduction}

NLTT 10480 (LHS 5070, LP 887-66) is a high proper-motion star (Luyten 1979, 1980) that was also listed as a white dwarf candidate in Luyten's white dwarf catalogue (Luyten 1977). Using an optical-infrared reduced proper-motion diagram (Salim $\&$ Gould 2002) and additional colourimetric criteria (Kawka et al. 2004), we selected NLTT 10480 for spectroscopic observations to investigate stellar properties such as the effective temperature $\left(T_{\text {eff }}\right)$, surface gravity $(\log g)$, chemical composition, and magnetic field strength, and to constrain its cooling age and mass.

The chemical composition of cool white dwarfs shows great diversity. Heavy elements, particularly calcium, are detected in close to a quarter of cool, hydrogen-rich (DA) white dwarfs, but with abundances well below solar (Zuckerman et al. 2003). The abundance of heavy elements decreases with white dwarf cooling ages mainly because of the increasing depth of the mixed convective layers in aging white dwarfs (Paquette et al. 1986; Koester 2009). Few examples of very cool $\left(T_{\text {eff }} \lesssim 5000 \mathrm{~K}\right)$ polluted white dwarfs (DAZ) are known, such as G 77-50 (WD 0322-019, Hintzen \& Strittmatter 1974; Sion et al. 1990), which is also harbouring a weak magnetic field (Farihi et al. 2011). New high-dispersion and high signal-to-noise ratio spectroscopic observations of faint high proper-motion stars are likely to contribute new objects to the current sample.

Heavy elements in the atmosphere of cool white dwarfs are almost certainly accreted from their immediate environment. Kilic et al. (2006) and Farihi et al. (2009) reported infrared observations of a sample of cool white dwarfs contaminated with heavy elements, and the authors noted an infrared-excess incidence of $\approx 10-20 \%$. This excess was attributed to debris

* Based on observations collected at the European Organisation for Astronomical Research in the Southern Hemisphere, Chile under programme ID 080.D-0521, 082.D-0750 and 086.D-0562. discs of temperatures ranging from a few hundred degrees to over $1000 \mathrm{~K}$. Some cool DAZ white dwarfs, such as G 174-74 (WD 0245+541), do not show an infrared excess (Debes et al. 2007), and the presence of heavy elements in cool white dwarfs with ages in excess of several billion years suggests, instead, the effect of episodic accretion from small asteroids rather than from a stable debris disc (Jura 2008). Therefore, the identification of new cool DAZ white dwarfs is of interest to constrain the phenomenon.

We present a first report on a programme aimed at identifying and characterizing new DAZ white dwarfs in the New Luyten Two-Tenths (NLTT) catalogue. Section 2 describes observations obtained at the European Southern Observatories (ESO) using the New Technology Telescope (3.6-m) and the Very Large Telescopes (VLTs). Section 3 presents our model atmosphere analysis including details of the model structures (Sect. 3.1), heavy element line opacities, and Zeeman effect on line profiles (Sect. 3.2), and measurements (Sect. 3.3). We summarize and discuss some implications of our results in Sect. 4.

\section{Observations}

We first observed NLTT 10480 with the focal reducer and low-dispersion spectrograph (FORS1) attached to the $8 \mathrm{~m}$ UT2 (Kueyen) at Paranal Observatory as part of our spectropolarimetric survey of white dwarfs. The purpose of the survey was to search for white dwarfs with weak magnetic fields. We used the 600B grism combined with a slit-width of 1 arcsec that provided a resolution of $6.0 \AA$. The spectra covered the range between 3780 and $6180 \AA$. The observations were conducted on UT 2007 Nov. 1 and consisted of a sequence of two consecutive exposures with an exposure time of $1360 \mathrm{~s}$ each. In the first exposure the Wollaston prism is rotated to $-45^{\circ}$ and it is followed by a second exposure with the Wollaston prism rotated to $+45^{\circ}$ 


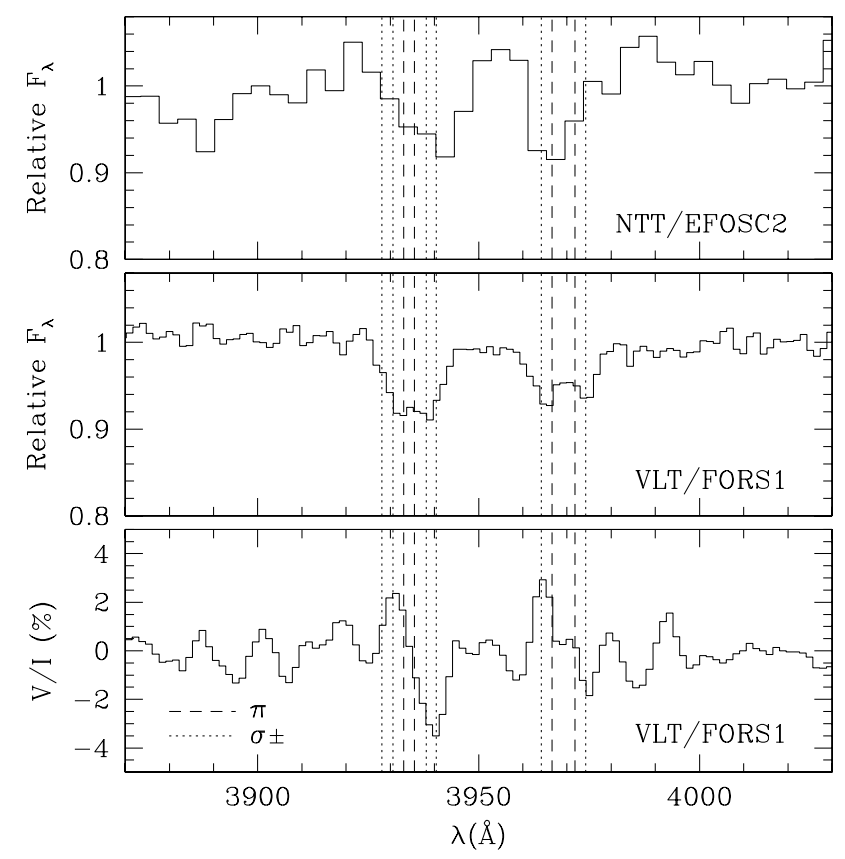

Fig. 1. Flux spectra of the Ca II H\&K lines in NLTT 10480 obtained with EFOSC2 (top) and FORS1 (middle), and circular polarization (bottom) spectrum obtained with FORS1. The dotted lines mark the position of the $\sigma$ components and the dashed lines mark the $\pi$ components (see Sect. 3.2.3)

from which we extracted the flux and circular polarization spectra.

Since the FORS1 spectra did not include $\mathrm{H} \alpha$, we obtained two additional low-dispersion spectra with the ESO Faint Object Spectrograph and Camera (EFOSC2) attached to the New Technology Telescope (NTT) at La Silla Observatory on UT 2008 Oct. 23. We used Grism 11 which has 300 lines per mm and a blaze wavelength of $4000 \AA$. The slit-width was set to 1 arcsec, which resulted in a spectral resolution of $\sim 14 \AA$. The exposure time of each spectrum was $1500 \mathrm{~s}$. Observations were carried out at the parallactic angle and were flux calibrated with the flux standard Feige 110.

Figure 1 compares the low-resolution spectrum of Ca II lines obtained with EFOSC2 to the higher resolution spectrum of FORS1. The flux and circular polarization spectra clearly show the $\sigma$ components, proving NLTT 10480 to be magnetic.

Finally, we obtained a set of echelle spectra of NLTT 10480 with the X-shooter spectrograph attached to the UT2 (Kueyen) at Paranal Observatory. The spectra were obtained at four epochs (UT 2010 Dec. 10, 2011 Jan. 9 and 26, and March 3). The 2011 Mar. 3 spectrum was of much poorer quality and was excluded from the analysis. The spectra were obtained with the slit-width set to $0.5,0.9$, and 0.6 arcsec for the UVB, VIS, and NIR arms, respectively. This set-up delivered a resolving power of 9100 for UVB, 8800 for VIS, and 6200 for NIR. The exposure time for each exposure was $2400 \mathrm{~s}$.

Table 1 summarizes our spectroscopic observations. The signal-to-noise ratio in the summed $\mathrm{X}$-shooter spectrum is $S / N \approx 53$ per binned-pixel at $4000 \AA$, while it is $\approx 120$ in the summed FORS1 spectrum and $\approx 65$ in the summed EFOSC2 spectrum at the same wavelength.

We searched for photometric measurements using VizieR. The Two Micron All Sky Survey (2MASS) listed infrared JHK
Table 1. Log of spectroscopic observations.

\begin{tabular}{lccc}
\hline \hline Instrument & UT date & Range & Note $^{1}$ \\
\hline VLT/FORS1 & 2007 Nov. 1 & $3780-6180 \AA$ & $\Delta \lambda \sim 6 \AA$ \\
NTT/EFOSC2 & 2008 Oct. 23 & $3680-7400 \AA$ & $\Delta \lambda \sim 14 \AA$ \\
VLT/X-shooter & 2010 Dec. 10 & $3000 \AA-2.5 \mu \mathrm{m}$ & $R \sim 9000$ \\
& 2011 Jan. 10 & & \\
& 2011 Jan. 26 & & \\
& 2011 Mar. 3 & & \\
\hline
\end{tabular}

Notes. ${ }^{(1)} \Delta \lambda \equiv F W H M, R \equiv \lambda / \Delta \lambda$.

Table 2. Photometry and astrometry.

\begin{tabular}{lcc}
\hline \hline Band & Measurement & Reference \\
\hline$V$ & $17.49 \pm 0.05$ & 1 \\
$J$ & $16.003 \pm 0.078$ & 2 \\
$H$ & $15.836 \pm 0.182$ & 2 \\
$K_{\mathrm{S}}$ & $16.529:$ & 2 \\
& & \\
RA (J2000.0) & 031712.08 & 3 \\
Dec (J2000.0) & -291134.33 & 3 \\
$(\mu, \theta)$ & $\left(0.496 \pm 0.008 \mathrm{mas} \mathrm{yr}^{-1}, 162^{\circ}\right)$ & 3 \\
& $\left(0.503 \pm 0.007 \mathrm{mas} \mathrm{yr}^{-1}, 159^{\circ}\right)$ & 4 \\
& $\left(0.49 \pm 0.01 \mathrm{mas} \mathrm{yr}^{-1}, 160^{\circ}\right)$ & 5 \\
\hline
\end{tabular}

References. (1) Johnson $V$, this work; (2) 2MASS (Skrutskie et al. 2006); (3) Revised NLTT (Salim \& Gould 2003); (4) SPM Catalog 2.0 (Platais et al. 1998); (5) Liverpool-Edinburgh High Proper Motion Catalogue (Pokorny et al. 2004).

magnitudes, but only the $J$ magnitude was of acceptable quality. We also used the acquisition images from the X-shooter observations to estimate a $V$ magnitude for NLTT 10480. We used 11 acquisition images of Feige 110 obtained between UT 2010 Dec. 10 and 2011 Jan. 1, and set the zero point. Next, we determined an average $V$ magnitude for NLTT 10480 using our five acquisition images. We employed the atmospheric extinction table of Patat et al. (2011). Table 2 lists the photometric measurements.

The SPM Catalog provides a photographic $B$ magnitude $(18.08 \pm 0.16)$. However, due to large uncertainties in the SPM and other photographic magnitudes, these are not as useful as our $V$ magnitude and the 2MASS $J$ magnitudes. The 2MASS $K$ magnitude is unreliable and the $H$ magnitude is uncertain. The index $B-V=0.59 \pm 0.17$ loosely constrains the temperature in the range $5100 \lesssim T_{\text {eff }} \lesssim 6700 \mathrm{~K}$, but the more precise index $V-J=1.49 \pm 0.09$ implies that NLTT 10480 is a cool white dwarf with $T_{\text {eff }} \approx 5050 \pm 150 \mathrm{~K}$ (see Table 3 and Sect. 3.1).

NLTT 10480 is also characterized by a large proper-motion of $\sim 0.5^{\prime \prime} \mathrm{yr}^{-1}$ (Table 2). The kinematical properties were determined using the proper-motion and the $\mathrm{X}$-shooter radial velocity measurement (see Sect. 4).

\section{Analysis}

\subsection{Model atmospheres and spectral syntheses}

We calculated a grid of model atmospheres for cool hydrogenrich white dwarfs. The models are in convective and radiative equilibrium with the total flux converged to better than $0.01 \%$ in all layers. The model grid covers the effective temperature range $4900 \leq T_{\text {eff }} \leq 6000 \mathrm{~K}$ in $100 \mathrm{~K}$ steps and the surface gravity range $7.5 \leq \log g \leq 8.5$ in steps of 0.25 dex. The adopted treatment of the convective energy transport and 
Table 3. Selected synthetic colours.

\begin{tabular}{lccc}
\hline \hline $\begin{array}{l}\log g \\
(\mathrm{cgs})\end{array}$ & $\begin{array}{c}T_{\text {eff }} \\
(\mathrm{K})\end{array}$ & $\begin{array}{c}B-V \\
(\mathrm{mag})\end{array}$ & $\begin{array}{c}V-J \\
(\mathrm{mag})\end{array}$ \\
\hline 7.5 & 4900 & 0.826 & 1.570 \\
& 5100 & 0.733 & 1.460 \\
& 5300 & 0.658 & 1.360 \\
& 5500 & 0.602 & 1.270 \\
& 5700 & 0.556 & 1.188 \\
8.0 & 4900 & 0.858 & 1.562 \\
& 5100 & 0.760 & 1.453 \\
& 5300 & 0.677 & 1.351 \\
& 5500 & 0.611 & 1.260 \\
8.5 & 5700 & 0.559 & 1.178 \\
& 4900 & 0.887 & 1.550 \\
& 5100 & 0.790 & 1.446 \\
& 5300 & 0.701 & 1.344 \\
& 5500 & 0.627 & 1.251 \\
& 5700 & 0.568 & 1.167 \\
\hline
\end{tabular}

pressure ionization effects are described in Kawka \& Vennes (2006), but further improvements to the models will be described in a forthcoming publication. All relevant species $\left(\mathrm{H}, \mathrm{H}^{+}, \mathrm{H}_{2}\right.$, $\mathrm{H}_{2}^{+}, \mathrm{H}_{3}^{+}$) are included in the statistical equilibrium equation. We employed the $\mathrm{H}_{3}^{+}$partition function of Neale \& Tennyson (1995). Electrons contributed by identifiable trace elements (e.g., calcium) are also included in the charge conservation equation, although the ionization of hydrogen atoms and molecules dominate the electron budget.

The model atmospheres include opacities caused by $\mathrm{H}$ bound-bound, bound-free and free-free transitions, $\mathrm{H}^{-}$boundfree and free-free transitions, $\mathrm{H}_{2}-\mathrm{H}_{2}$ collision-induced absorption (CIA, Borysow et al. 1997, 2001), and the $\mathrm{H}_{2}-\mathrm{H}$ and $\mathrm{H}-\mathrm{H}$ collision-induced absorptions in the far Ly $\alpha$ wing (see Kowalski \& Saumon 2006) using opacity tables from Rohrmann et al. (2011). Finally, the $\mathrm{H}_{2}$ and $\mathrm{H}$ Rayleigh scattering are included along with electron scattering.

Synthetic colours as well as detailed hydrogen and heavy element line profiles are computed using the model structures. Table 3 lists some photometric properties of the cool models. The colour indices at shorter wavelengths are effected by the Ly $\alpha$ collision-induced absorptions. Allard et al. (2008) calculated new $\mathrm{H} \alpha$ line profiles including self-broadening effects and found a half-width at half maximum $(\mathrm{HWHM}) \approx 40 \%$ larger than estimated by Ali \& Griem $(1965,1966)$ and comparable to the calculations of Barklem et al. (2000a). We adopted the $\mathrm{H} \alpha$ HWHM from Allard et al. (2008) and the $\mathrm{H} \beta$, and $\mathrm{H} \gamma$ crosssections and velocity parameters, converted into HWHM, from Barklem et al. (2000a). We describe the heavy element line profiles in the following section.

\subsection{Neutral and ionized line profiles}

\subsubsection{Line broadening}

The dominant broadening mechanism is collision with hydrogen atoms. We employed the coefficients of Barklem et al. (2000b), where the full-width at half-maximum $(F W H M)$ of the Lorentzian profiles is given by

$\frac{w}{n(\mathrm{HI})}=\left(\frac{T}{10^{4} \mathrm{~K}}\right)^{(1-\alpha) / 2} \Gamma \operatorname{rad~s}^{-1} \mathrm{~cm}^{3}$,

where $\log \Gamma=-7.562$ for $\mathrm{Ca} \mathrm{I} \lambda 4226$ and -7.76 for Ca II H\&K at $T=10000 \mathrm{~K}$, and $\alpha=0.238$ for $\mathrm{Ca} \mathrm{I} \lambda 4226$ and 0.223 for
Ca II H\&K. Although the adopted broadening parameters do not include the effect of hydrogen molecules, these provide $>50 \%$ of the gas pressure in some layers. Following the approximate treatment of Kurucz \& Avrett (1981), the broadening parameter $\Gamma \propto n_{\mathrm{H}}+0.85 n\left(\mathrm{H}_{2}\right)$, and, therefore, hydrogen molecules may contribute to the total line width. We found that neither $\mathrm{Ca}$ I or $\mathrm{Ca}$ II equivalent widths are significantly affected in models at $T_{\text {eff }}=5400 \mathrm{~K}$, but abundances inferred from Ca I $\lambda 4226$ may be underestimated by a factor of $\sim 2$ in models at $4900 \mathrm{~K}$.

\subsubsection{Zeeman effect}

Fundamentals of stellar line formation in the presence of a magnetic field are described by Unno (1956) and Martin \& Wickramasinghe (1981), who also describe significant magnetooptical effects. In particular, Unno (1956) showed that the effect of field inclination with respect to the line-of-sight on the relative intensity of $\sigma$ and $\pi$ components reaches a maximum at an angle of $55^{\circ}$. Moreover, Martin \& Wickramasinghe (1981) showed that taking into account magneto-optical effects may enhance the depth of the $\pi$ components upon certain conditions.

Kemic (1975) studied the quadratic Zeeman effect for the $\mathrm{Ca}$ II $\mathrm{H}$ and $\mathrm{K}$ lines and showed that the linear Zeeman effect at fields of $15 \mathrm{MG}$ is still dominant.

The $\mathrm{Ca}$ II $\mathrm{H}$ and $\mathrm{K}$ lines are the result of transitions between the ground state with $J, L, S=\frac{1}{2}, 0, \frac{1}{2}$, and the excited states with $J, L, S=\frac{1}{2}, 1, \frac{1}{2}$ and $\frac{3}{2}, 1, \frac{1}{2}$, respectively, where $J$ is the total angular momentum, $L$ is the orbital angular momentum and $S$ is the spin angular momentum. The $\mathrm{Ca} \mathrm{I} \lambda 4226$ line is the result of transitions between the ground state with $J, L, S=0,0,0$ and the excited state with $J, L, S=1,1,0$.

The levels are split by a magnetic field into $2 J+1$ components defined by the magnetic quantum number $m=-J, \ldots, J$ :

$\Delta \lambda=\frac{e B \lambda^{2}}{4 \pi m_{\mathrm{e}} c^{2}}\left(g_{\mathrm{l}} m_{1}-g_{\mathrm{u}} m_{\mathrm{u}}\right) \approx 4.67 \times 10^{-7} \lambda^{2} B\left(g_{\mathrm{l}} m_{1}-g_{\mathrm{u}} m_{\mathrm{u}}\right)$,

where $\lambda$ is the wavelength in $\AA, B$ is the magnetic field in MG, $e$ is the electron charge, $m_{\mathrm{e}}$ is the electron rest mass and $c$ is the speed of light. The Landé factor and the magnetic quantum number of the upper and lower levels are given by $g_{\mathrm{u}}, m_{\mathrm{u}}$ and $g_{1}, m_{1}$, respectively. Landé factors for calcium and other elements except iron were calculated assuming LS coupling:

$g=1+\frac{J(J+1)-L(L-1)+S(S+1)}{2 J(J+1)}$.

For the $4 \mathrm{~S}_{1 / 2}$ state of Ca II, the experimentally determined $g$ factor of 2.00225664 (Tommaseo et al. 2003) agrees with the theoretically calculated factor based on the LS coupling scheme. Similarly, for the $3 p{ }^{2} \mathrm{P}_{3 / 2}$ state of AlI, the experimentally determined $g$ factor of $1.33474 \pm 0.00005$ (Martin et al. 1968) agrees with the LS coupling scheme calculated factor. For iron, the Landé factors were obtained from the Vienna Atomic Line Database (VALD) ${ }^{1}$.

The permitted transitions are defined by $\Delta m=0, \pm 1$, where $\Delta m=0$ defines the $\pi$ components and $\Delta m= \pm 1$ the $\sigma$ components.

${ }^{1}$ http://vald.astro.univie.ac.at (Kupka et al. 2000). 
Table 4. Zeeman splitting at $B=0.513 \mathrm{MG}$ ( Ca I and Ca II).

\begin{tabular}{|c|c|c|c|c|c|c|c|}
\hline \multicolumn{3}{|c|}{ Lower level } & \multicolumn{3}{|c|}{ Upper level } & \multirow{2}{*}{$\begin{array}{c}\lambda \\
(\AA)\end{array}$} & \multirow[t]{2}{*}{ Rel. Int. } \\
\hline$J$ & $g$ & $m$ & $J$ & $g$ & $m$ & & \\
\hline \multirow{7}{*}{$1 / 2$} & & & \multicolumn{3}{|c|}{ Ca II $\lambda 3933.663$} & & \\
\hline & 2 & $-1 / 2$ & $3 / 2$ & $4 / 3$ & $1 / 2$ & 3927.482 & $1 / 16$ \\
\hline & & $1 / 2$ & & & $3 / 2$ & 3929.955 & $3 / 16$ \\
\hline & & $-1 / 2$ & & & $-1 / 2$ & 3932.427 & $1 / 4$ \\
\hline & & $1 / 2$ & & & $1 / 2$ & 3934.899 & $1 / 4$ \\
\hline & & $-1 / 2$ & & & $-3 / 2$ & 3937.371 & $3 / 16$ \\
\hline & & $1 / 2$ & & & $-1 / 2$ & 3939.844 & $1 / 16$ \\
\hline \multirow{5}{*}{$1 / 2$} & & & \multicolumn{3}{|c|}{ Сa II $\lambda 3968.469$} & & \\
\hline & 2 & $-1 / 2$ & $1 / 2$ & $2 / 3$ & $1 / 2$ & 3963.436 & $1 / 4$ \\
\hline & & $-1 / 2$ & & & $-1 / 2$ & 3965.953 & $1 / 4$ \\
\hline & & $1 / 2$ & & & $1 / 2$ & 3970.985 & $1 / 4$ \\
\hline & & $1 / 2$ & & & $-1 / 2$ & 3973.502 & $1 / 4$ \\
\hline \multirow{4}{*}{0} & & & \multicolumn{3}{|c|}{ Ca I $\lambda 4226.728$} & & \\
\hline & 0 & 0 & 1 & 1 & 1 & 4222.446 & $1 / 4$ \\
\hline & & 0 & & & 0 & 4226.728 & $1 / 2$ \\
\hline & & 0 & & & -1 & 4231.010 & $1 / 4$ \\
\hline
\end{tabular}

\subsubsection{Opacity and line intensities}

The relative intensities of the Zeeman components are computed following Condon \& Shortley (1963). They found for the transitions where $\Delta J=0$ and $\Delta m=0$ ( $\pi$ components $)$

$I \propto m^{2}$,

or where $\Delta m= \pm 1(\sigma$ components $)$

$I \propto \frac{1}{4}(J \mp m)(J \mp m+1)$.

Similarly, they found for transitions where $J \rightarrow J+1$ and for the $\pi$ components

$I \propto(J+1)^{2}-m^{2}$,

and for the $\sigma$ components

$I \propto \frac{1}{4}(J \pm m+1)(J \pm m+2)$.

Finally, for transitions where $J \rightarrow J-1$, the relative intensities are for the $\pi$ components

$I \propto J^{2}-m^{2}$

and for the $\sigma$ components

$I \propto \frac{1}{4}(J \mp m)(J \mp m-1)$.

Table 4 lists the total angular momenta, Landé factors, magnetic quantum numbers for the various transitions of calcium in a magnetic field of $0.513 \mathrm{MG}$. The relative intensities for the different components are also provided.

The observed relative intensities of the $\pi$ and $\sigma$ components will also vary as a function of the angle between the magnetic field lines and the line of sight (Unno 1956; Martin \& Wickramasinghe 1981). The monochromatic opacity of the $\pi$ components depends on the angle $\psi$ between the magnetic field axis and the line of sight:

$\frac{\chi_{\pi}(\psi)}{\chi_{\pi}\left(90^{\circ}\right)}=\sin ^{2} \psi$
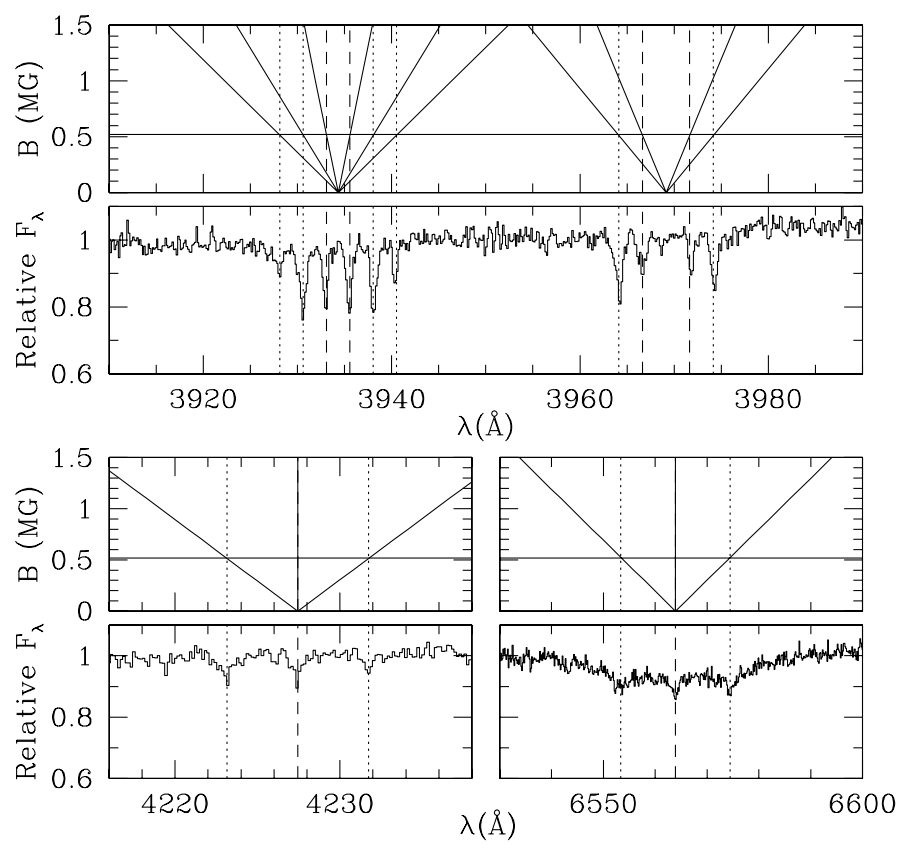

Fig. 2. Linear Zeeman splitting of Ca II (H and K) (top), Ca I $4226 \AA$ (bottom left), and $\mathrm{H} \alpha$ (bottom right) lines at $B=0.519 \mathrm{MG}$ compared to the observed Zeeman splitting in the spectrum of NLTT 10480. The dotted lines indicate the $\sigma$ components and dashed lines indicate the $\pi$ components.

Similarly, the monochromatic opacity of the $\sigma$ components vary as

$\frac{\chi_{\sigma}(\psi)}{\chi_{\sigma}\left(90^{\circ}\right)}=\left(1+\cos ^{2} \psi\right)$,

where the opacities $\chi_{\pi}\left(90^{\circ}\right)$ and $\chi_{\sigma}\left(90^{\circ}\right)$ are given at $\psi=$ $90^{\circ}$ and, as shown earlier, are calculated following Condon \& Shortley (1963). For example, for hydrogen Balmer lines or $\mathrm{Ca}$ I $\lambda 4226$ we have that

$\chi_{\pi}\left(90^{\circ}\right)=\frac{1}{2} \chi_{0}$, and $\chi_{\sigma}\left(90^{\circ}\right)=\frac{1}{4} \chi_{0}$.

Figure 2 shows the observed linear Zeeman splitting of $\mathrm{Ca}$ II $\mathrm{H} \& \mathrm{~K}, \mathrm{Ca} \mathrm{I} \lambda 4226 \AA$, and $\mathrm{H} \alpha$ lines obtained with the X-shooter spectrograph and the predicted line positions as a function of the magnetic field strength. Interestingly, the observed $\pi$ components for both $\mathrm{Ca}$ II lines appear weaker than the $\sigma$ components showing the effect of inclination of the field with respect to the line-of-sight. No significant variation in the line positions or intensities were noted between the three usable exposures.

We fitted the ratio of the $\mathrm{Ca}$ II $\pi$ and $\sigma$ line equivalent widths varying the inclination and determined $\psi=60 \pm 3^{\circ}$. However, including magneto-optical effects would likely decrease the measured inclination.

\subsection{Magnetic field and atmospheric parameters}

We determined the averaged surface magnetic field of NLTT 10480 using $\mathrm{H} \alpha$ and the calcium lines. We first measured the centroids of the Zeeman components of Ca II, Ca I and $\mathrm{H} \alpha$, where the spectrum was already adjusted to the solar system barycentre. We then fitted these lines to the predicted line positions by varying the magnetic field strength (assumed constant over the surface) and the velocity and by minimizing the $\chi^{2}$. For calcium, we determined a surface-averaged magnetic field of 

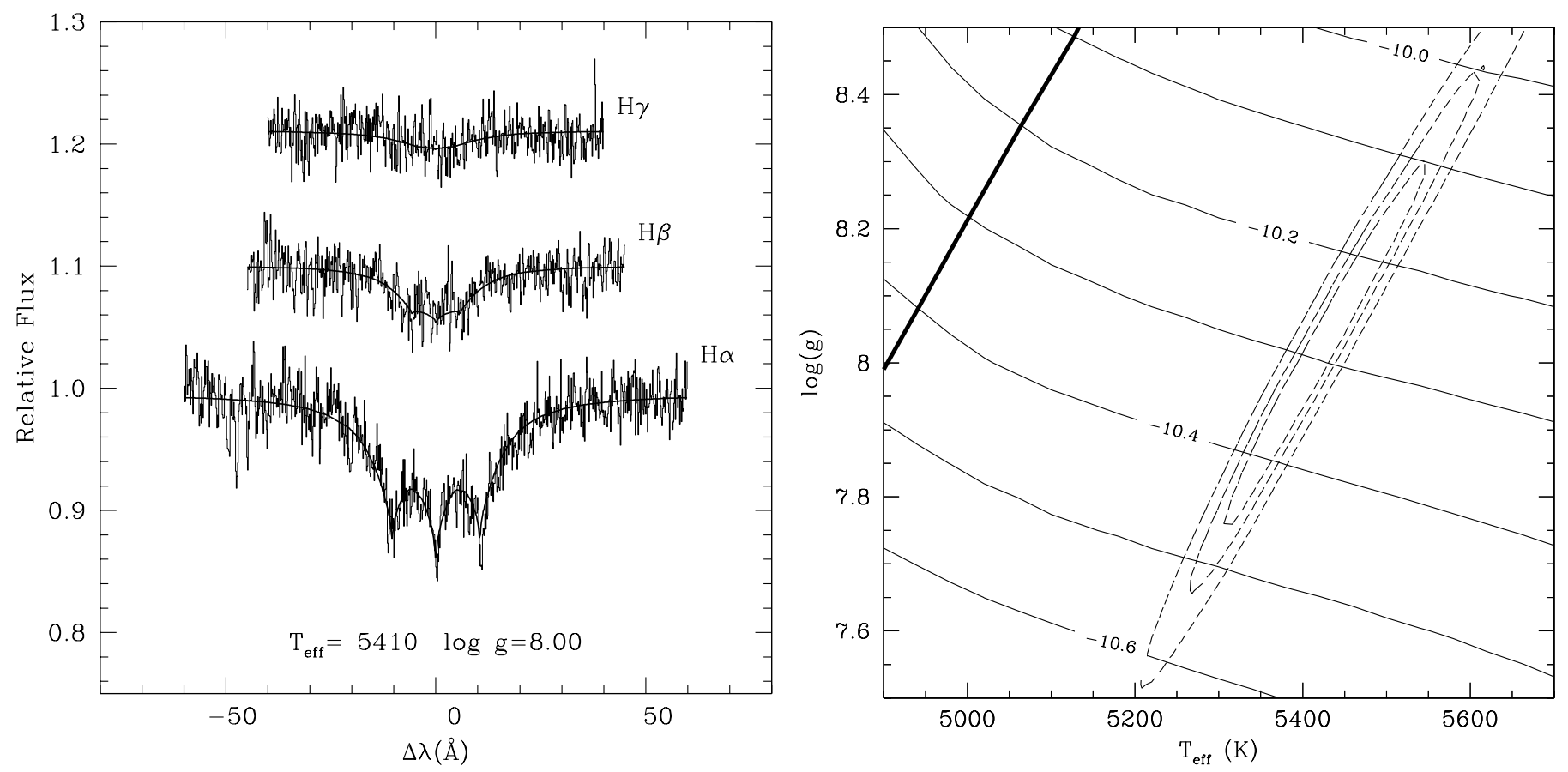

Fig. 3. Balmer lines $(\mathrm{H} \alpha$ to $\mathrm{H} \gamma)$ and best-fitting model $\left(T_{\mathrm{eff}}=5410 \mathrm{~K}, \log g=8.0\right)$ showing prominent Zeeman-split $\mathrm{H} \alpha$ line (left panel), and (right panel) $\chi^{2}$ contours at 66,90 , and $99 \%$ confidence (dashed lines) in the $\left(T_{\mathrm{eff}}, \log g\right.$ ) plane. The measured calcium abundance from $\mathrm{Ca}$ II lines $(\log n(\mathrm{Ca}) / n(\mathrm{H}))$ is drawn (full lines) as a function of the assumed temperature and surface gravity and labelled with the logarithm of the abundance. The thick line shows the range of temperature and surface gravity where the abundance measurements based on Ca I or Ca II are consistent.

$B_{\mathrm{S}}=0.513 \pm 0.005 \mathrm{MG}$ with a velocity of $v=50.8 \pm 3.0 \mathrm{~km} \mathrm{~s}^{-1}$. Using $\mathrm{H} \alpha$, we obtained $B_{\mathrm{s}}=0.525 \pm 0.005 \mathrm{MG}$ and $v=$ $51.8 \pm 3.0 \mathrm{~km} \mathrm{~s}^{-1}$. The measurements are consistent within $2 \sigma$ and taking the average we found $B_{\mathrm{s}}=0.519 \pm 0.004$ and $v=51.3 \pm 2 \mathrm{~km} \mathrm{~s}^{-1}$. Subtracting the gravitational redshift $\left(v_{\mathrm{gr}}=29.3_{-4.8}^{+34.2} \mathrm{~km} \mathrm{~s}^{-1}\right)$ of the white dwarf from the measured velocity results in the actual line of sight velocity of the star $v_{\mathrm{r}}=22_{-5}^{+34} \mathrm{~km} \mathrm{~s}^{-1}$.

In principle, the effective temperature and surface gravity may be constrained simultaneously by fitting the Balmer line profiles. The strong $\mathrm{H} \alpha$ and weaker $\beta$ show the effect of Zeeman line splitting, while $\mathrm{H} \gamma$ is extremely weak. Figure 3 shows the best-fitting model to the Balmer lines that includes an approximate treatment of line opacities in a weak magnetic field. The $99 \%$ confidence contour corresponds to an uncertainty of $200 \mathrm{~K}$ in the temperature, and 0.5 dex in the logarithm of the surface gravity. The errors are statistical only and do not take into account possible systematic errors in the modelling of the line profiles.

\subsubsection{Abundance of heavy elements}

We determined the calcium abundance by fitting the Ca II line profiles using $\chi^{2}$ minimization techniques where we varied the calcium abundance at each grid point in the $\left(T_{\text {eff }}, \log g\right)$ plane while fixing the magnetic field strength at $0.519 \mathrm{MG}$ (Sect. 3.3). Figure 3 shows the resulting abundance map: the calcium abundance and its uncertainty depend on the adopted atmospheric parameters $T_{\text {eff }}$ and $\log g$.

Figure 4 shows best-fitting models to the calcium lines at $T_{\text {eff }}=5400(\log g=8)$ and at a lower temperature of $4900 \mathrm{~K}(\log g=8)$. The calcium abundance varies between $\log n(\mathrm{Ca}) / n(\mathrm{H})=-10.3$ (highest temperature) and -10.45 (lowest temperature), but the calcium ionization balance favours a lower temperature than estimated with the Balmer lines (Fig. 3). The discrepancy is partially resolved by increasing the metallicity of the atmosphere. Using a set of heavy elements with low first-ionization potentials and higher solar abundances including $\mathrm{C}, \mathrm{N}$, and $\mathrm{O}$, then $\mathrm{Na}$ to $\mathrm{Si}$, and, finally, $\mathrm{K}$ to $\mathrm{Cu}$, we found that a high metallicity of $0.03 \times$ solar would restore the calcium ionization balance with an abundance of $\log n(\mathrm{Ca}) / n(\mathrm{H})=-10.2$. However, a much lower abundance of heavy elements is present in the atmosphere of NLTT 10480.

Figure 5 shows the predicted location of the strongest lines of Fe I, Si I, Al I and Na I in the X-shooter spectrum. We calculated the position of the Zeeman-split lines for these elements assuming a magnetic field of 0.519 MG. For aluminium, some weak lines appear to match the predicted positions. However, the putative Al I $\lambda 3967.8532$ component should be accompanied by stronger $\lambda 3957.7353$ and $\lambda 3960.2458$ components that are not clearly identified. Another possible identification for this feature is an interstellar $\mathrm{Ca} \mathrm{H}$ line, although $\mathrm{Ca} \mathrm{K}$ is not detected in the spectrum. Spectra with higher signal-to-noise ratios and better resolution are needed to clarify this identification.

We estimated sodium, aluminium, silicon and iron abundance upper limits using the spectral ranges covered in Fig. 5. We found $\log n(\mathrm{Na}) / n(\mathrm{H}), \log n(\mathrm{Al}) / n(\mathrm{H})$, and $\log n(\mathrm{Fe}) / n(\mathrm{H}) \lesssim-9.3$, while $\log n(\mathrm{Si}) / n(\mathrm{H}) \lesssim-8.7$. The abundances relative to solar range from $2 \times 10^{-5}$ to $3 \times 10^{-4}$ times solar, or a few orders of magnitude below the level required to significantly increase the electron density and alter the calcium ionization balance.

\section{Summary and discussion}

We found that the high proper-motion star NLTT 10480 is a rare example of cool white dwarfs with trace heavy elements and a weak magnetic field revealed in both the $\mathrm{H} \alpha$ circular polarization 


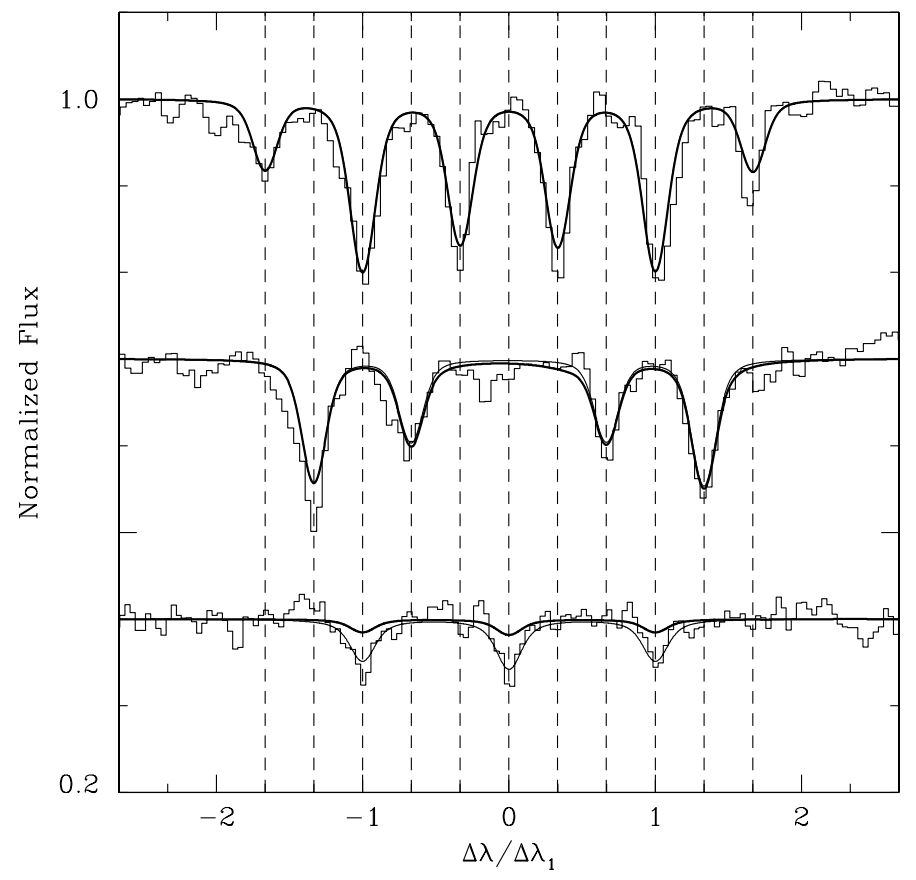

Fig. 4. X-shooter spectrum of the Ca I (bottom) and Ca II (H \& K: middle and top, respectively) lines and best-fitting models at $(\log g=8)$ $T_{\text {eff }}=5400 \mathrm{~K}$ and $\log n(\mathrm{Ca}) / n(\mathrm{H})=-10.3$ (thick lines), and $T_{\text {eff }}=$ $4900 \mathrm{~K}$ and $\log n(\mathrm{Ca}) / n(\mathrm{H})=-10.45$ (thin lines). The wavelength scale is normalized to $\Delta \lambda_{1} \equiv 4.67 \times 10^{-7} \lambda^{2} B$, where $B=0.513 \mathrm{MG}$. Therefore, the line positions match the factors $g_{1} m_{1}-g_{\mathrm{u}} m_{\mathrm{u}}$ listed in Table 4 (vertical dashed lines). All models assume $\psi=60^{\circ}$.

spectrum and Zeeman line-splitting. Other examples of this class of white dwarfs are the DZ white dwarfs LHS 2534 (Reid et al. 2001) and G 165-7 (Dufour et al. 2006), the DAZ white dwarfs G 77-50 (Farihi et al. 2011) and possibly LTT 8381 (Koester et al. 2009). Based on independent diagnostics (Table 5), we estimated a temperature of $T_{\text {eff }}=5200 \pm 200 \mathrm{~K}$ and a surface gravity close to $\log g=8$. However, we noted systematic differences in temperature measurements based on the calcium ionization ratio, the colour index, and the Balmer line profiles amounting to $\sim 400 \mathrm{~K}$. The weaker $\mathrm{Ca}$ I lines favour a lower temperature than estimated using Balmer lines alone. The temperature measured with the $V-J$ colour index also favours a lower temperature. We found that increasing the heavy-element contribution to the electron density helps restore the calcium ionization balance, but we also found that the required abundance exceeds upper limits on the abundance of $\mathrm{Na}, \mathrm{Al}, \mathrm{Si}$, and Fe by a few orders of magnitude. We are left with the possibility that subtle effects on line formation (broadening parameters, magnetic-optical, ...) caused by the magnetic field may influence temperature measurements based on Balmer line profiles.

Although our modelling of the hydrogen line profiles takes into account the effect of inclination, we neglected the magnetooptical effects and only approximated the full solution of the radiative transfer equations that ought to include all Stokes parameters (see Martin \& Wickramasinghe 1981). The effect of this approximation on the determination of the stellar parameters using Balmer line profile fitting may well amount to a few hundred degrees.

Fortunately, because ionized calcium is the dominant species, the abundance of calcium based on $\mathrm{Ca}$ II lines is not sensitive to temperature. On the other hand, as we have
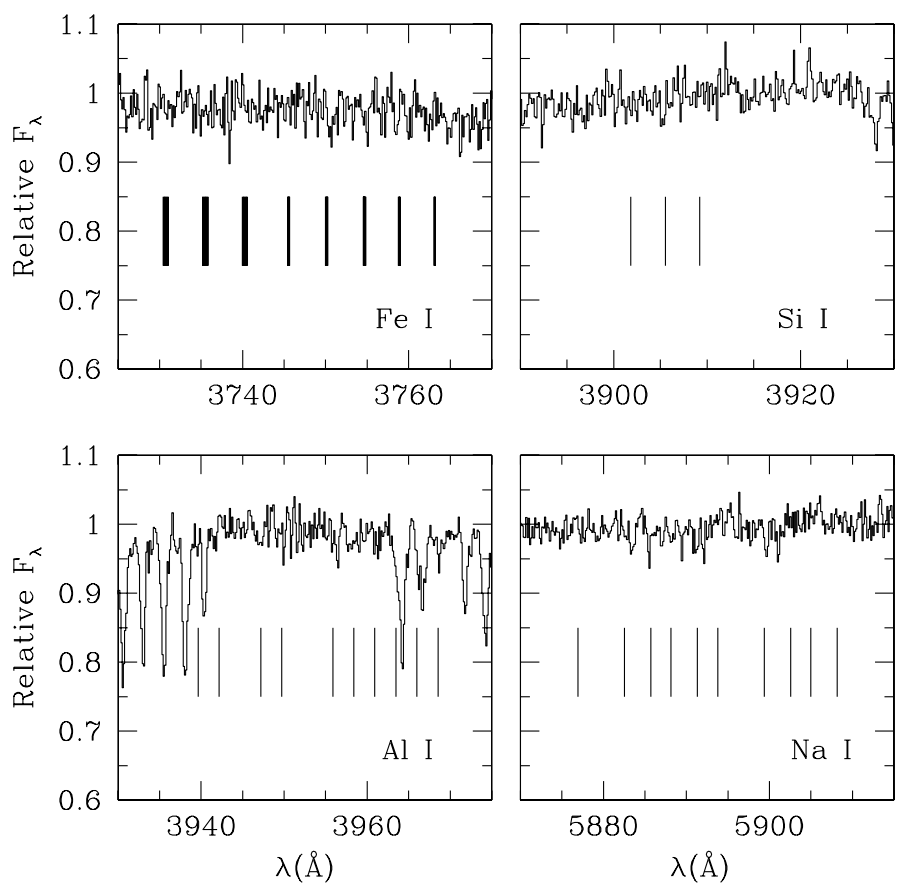

Fig. 5. Spectra in the vicinity of the strongest Fe I (3734.8638, 3749.4851, and 3758.2330 ̊), Si I (3905.523 ̊), Al I (3944.0060 and $3961.5200 \AA$ ), and Na I (5889.951 and 5895.924 Å) lines compared to the predicted Zeeman-split line positions assuming a magnetic field of $0.519 \mathrm{MG}$.

Table 5. Properties of NLTT 10480.

\begin{tabular}{lcc}
\hline \hline Parameter & Measurement & Note \\
\hline$B_{\mathrm{S}}$ & $0.519 \pm 0.004 \mathrm{MG}$ & 1 \\
$v_{\mathrm{r}}$ & $22_{-5}^{+34} \mathrm{~km} \mathrm{~s}^{-1}$ & 1 \\
$T_{\text {eff }}$ & $5050 \pm 150 \mathrm{~K}$ & 2 \\
& $5400 \pm 200 \mathrm{~K}$ & 3 \\
$\log g$ & $8.0 \pm 0.5$ & 3 \\
Abundance & $\log n(\mathrm{Na}) / n(\mathrm{H}) \lesssim-9.3$ & 4 \\
& $\log n(\mathrm{Al}) / n(\mathrm{H}) \lesssim-9.3$ & 4 \\
& $\log n(\mathrm{Si}) / n(\mathrm{H}) \lesssim-8.7$ & 4 \\
& $\log n(\mathrm{Ca}) / n(\mathrm{H})=-10.3 \pm 0.3$ & 4 \\
$d$ & $\log n(\mathrm{Fe}) / n(\mathrm{H}) \lesssim-9.3$ & 4 \\
& $33_{-14}^{+9} \mathrm{pc}$ & 5 \\
$U, V, W$ & $46_{-31}^{+16},-66_{-31}^{+29},-3_{-33}^{+7} \mathrm{~km} \mathrm{~s}^{-1}$ & 6 \\
\hline
\end{tabular}

Notes. (1) Sect. 3.2; (2) $V-J$, Sect. 2; (3) Balmer lines, Sect. 3.3; (4) Sect. 3.3 .1 ; (5) Based on apparent and absolute $V$ magnitudes (Sect. 4); (6) Based on proper-motion and the estimated distance.

demonstrated, it does show a mild dependence on surface gravity because a higher electron pressure favours neutral calcium.

Adopting conservative error bars for the temperature and surface gravity we calculated an absolute $V$ magnitude $M_{V}=$ $14.9_{-0.6}^{+0.9}$ using the mass-radius relations of Benvenuto \& Althaus (1999). The distance modulus implies a photometric distance $d=33_{-14}^{+9}$ pc. The object is relatively old with a cooling age $t_{\text {cool }}=3.5-8.0 \mathrm{Gyr}$, but with an uncertain mass $\left(0.35-0.91 M_{\odot}\right)$. We determined the Galactic velocity vector $U V W$ (Table 5) using our distance estimate and radial velocity measurement (Sect. 3.3), and published proper-motion. We employed the algorithm of Johnson \& Soderblom (1987). The kinematics imply membership to the old thin disk (Sion et al. 1988) consistent with the upper range of our age estimate. 
The acquisition of broadband $U B V$ and $J H K$ photometry and of a parallax measurement should help determine the atmospheric parameters more precisely. The stellar radius, hence surface gravity measurement would be improved with a parallax measurement. Accordingly, the error on the calcium abundance measurement would be reduced. Accurate $J H K$ photometry would also allow us to investigate possible infrared excess and the presence of a debris disc.

The DAZ white dwarfs G 77-50 and G 174-74 are part of a survey including the coolest known DAZ white dwarfs (Zuckerman et al. 2003). In cool convective white dwarfs, heavy elements diffuse below the mixed convective layers, and their presence in white dwarf atmospheres is transitory. Koester \& Wilken (2006) estimated the diffusion time-scale for various heavy elements. The accretion rate required to sustain a given mass fraction $X$ in the atmosphere is given by

$\dot{M}_{\mathrm{acc}}=\frac{X}{X_{\mathrm{acc}}} \frac{M_{\mathrm{cvz}}}{\tau}$,

where $M_{\mathrm{cvz}}$ is the mass of the convection zone and $\tau$ the diffusion time-scale at the bottom of the convection zone, where diffusion is allowed to take place. The ratio $X / X_{\text {acc }}$ is the ratio of the measured mass-fraction to the accreted mass fraction. Therefore, the mass accretion rate of any particular element is

$X_{\mathrm{acc}} \dot{M}_{\mathrm{acc}}=X \frac{M_{\mathrm{cvz}}}{\tau}$

Adopting, in the appropriate temperature range, a value for the slow-varying ratio $M_{\mathrm{cvz}}$ to diffusion time scale of $\log \left(M_{\mathrm{cvz}} / \tau_{\mathrm{Ca}}\right) \approx-11.5$ in units of $M_{\odot} \mathrm{yr}^{-1}$ or $=14.3$ in units of $\mathrm{g} \mathrm{s}^{-1}$ (Koester \& Wilken 2006), we estimated the mass accretion rate of calcium (in $\mathrm{g} \mathrm{s}^{-1}$ ) to be

$\log \left(X_{\mathrm{acc}, \mathrm{Ca}} \dot{M}_{\mathrm{acc}}\right)=\log \left(M_{\mathrm{cvz}} / \tau_{\mathrm{Ca}}\right)+\log X_{\mathrm{Ca}}=5.60$,

where $X_{\mathrm{Ca}} \approx\left[A_{\mathrm{Ca}} n(\mathrm{Ca})\right] /\left[A_{\mathrm{H}} n(\mathrm{H})\right]=2 \times 10^{-9}$, and $A_{\mathrm{Ca}}$ and $A_{\mathrm{H}}$ are the atomic weights. Assuming calcium is accreted as part of a solar-composition flow, the total mass accretion rate (including hydrogen) is $5 \times 10^{9} \mathrm{~g} \mathrm{~s}^{-1}$ or $8 \times 10^{-17} M_{\odot} \mathrm{yr}^{-1}$.

Our measured error on the calcium abundance of $\pm 0.3 \mathrm{dex}$ translates into a similar error on the calculated accretion rate onto the white dwarf surface. The true error may well be much larger. Koester (2009) considers that the application of the mixing-length theory to the structure of convection zones may underestimate the mass of the mixed layers by orders of magnitude. The effect of "under"-shooting below the convection zone may affect diffusion time-scale estimates. Therefore, the precision claimed in measuring abundance of parent bodies may be over-estimated.

Farihi et al. (2011) propose that the model of Potter \& Tout $(2010)^{2}$ for the presence of a magnetic field in post-common envelope (CE) binaries could also be applied to $\mathrm{CE}$ episodes with planetary rather than stellar secondary components. Whether the magnetic field is acquired during such a process, or whether it is a fossil field cannot be ascertained for individual objects but rather from population studies (see, e.g., Kawka \& Vennes 2004; Kawka et al. 2007; Wickramasinghe \& Ferrario 2005). In the case of NLTT 10480, which is old (>3.5 Gyr) with relatively short diffusion time-scales, the present-day metallicity is not linked to the $\mathrm{CE}$ event that potentially generated the magnetic field, but more likely to a recent accretion event.

\footnotetext{
${ }^{2}$ See also Nordhaus et al. (2011).
}

A low incidence of planetary systems would imply a low incidence of weak magnetic fields ( $B \lesssim 1 \mathrm{MG}$ ). Current data indicate a low incidence of weak magnetic fields, and Kawka et al. (2007) found that 6 out of 53 local white dwarfs $(d \leq 20 \mathrm{pc})$ observed with sufficient accuracy to unveil fields weaker than $1 \mathrm{MG}$ were found to harbour such a low field. Moreover, Kawka $\&$ Vennes (2004) found that low-field white dwarfs lack progenitors, a gap that could be filled with the CE-mechanism.

Early results from the Kepler survey also indicate a low incidence of very large planets in short-period orbits ( $P \lesssim 50$ days, or $a \lesssim 0.2 \mathrm{AU})$ that are likely to trigger the field-generating CE events postulated by Farihi et al. (2011) based on the model of Potter \& Tout (2010). Planets with sizes ranging from 8 to $32 R_{\oplus}\left(=0.7-2.9 R_{\mathrm{J}}\right)$ may occur within 0.25 AU of solar-type stars with a frequency of $1.3 \%$ (Howard et al. 2011). Borucki et al. (2011) quote a similar fraction for Jupiter-sized or larger planets within 0.2 AU based on the first data set from Kepler. Both studies noted a declining occurrence with increasing separation. Overall, large planets that are likely to participate in a CE phase may surround a few percents of white dwarf progenitors and generate, as observed, a similar percentage of low-field white dwarfs.

Acknowledgements. S.V. and A.K. are supported by GA AV grant numbers IAA300030908 and IAA301630901, respectively, and by GA ČR grant number P209/10/0967. A.K. also acknowledges support from the Centre for Theoretical Astrophysics (LC06014). We thank the anonymous referee and R. Napiwotzki for several comments that improved the paper. This research has made use of the VizieR catalogue access tool, CDS, Strasbourg, France. This publication makes use of data products from the Two Micron All Sky Survey, which is a joint project of the University of Massachusetts and the Infrared Processing and Analysis Center/California Institute of Technology, funded by the National Aeronautics and Space Administration and the National Science Foundation.

\section{References}

Ali, A. W., \& Griem, H. R. 1965, Phys. Rev., 140, 1044 Ali, A. W., \& Griem, H. R. 1966, Phys. Rev., 144, 366

Allard, N. F., Kielkopf, J. F., Cayrel, R., \& van't Veer-Menneret, C. 2008, A\&A, 480, 581

Barklem, P. S., Piskunov, N., \& O’Mara, B. J. 2000a, A\&A, 363, 1091

Barklem, P. S., Piskunov, N., \& O'Mara, B. J. 2000b, A\&AS, 142, 467

Benvenuto, O. G., \& Althaus, L. G. 1999, MNRAS, 303, 30

Borucki, W. J., Koch, D. G., Basri, G., et al. 2011, ApJ, 728, 117

Borysow, A., Jørgensen, U. G., \& Zheng, C. 1997, A\&A, 324, 185

Borysow, A., Jørgensen, U. G., \& Fu, Y. 2001, JQSRT, 68, 235

Condon, E. U., \& Shortley, G. H. 1963 (Cambridge University Press)

Debes, J. H., Sigurdsson, S., \& Hansen, B. 2007, AJ, 134, 1662

Dufour, P., Bergeron, P., Schmidt, G. D., et al. 2006, ApJ, 651, 1112

Farihi, J., Jura, M., \& Zuckerman, B. 2009, ApJ, 694, 805

Farihi, J., Dufour, P., Napiwotzki, R., \& Koester, D. 2011, MNRAS, 413, 2559

Hintzen, P., \& Strittmatter, P. A. 1974, ApJ, 193, L111

Howard, A. W., Marcy, G. W., Bryson, S. T., et al. 2011, ApJ, submitted [arXiv: 1103.2541]

Johnson, D. R. H., \& Soderblom, D. R. 1987, AJ, 93, 864

Jura, M. 2008, AJ, 135, 1785

Kawka, A., \& Vennes, S. 2004, in A-Star Puzzle (Cambridge University Press), ed. J. Zverko, J. Ziznovsky, S. J. Adelman, \& W. W. Weiss, Proc. IAU Symp., 224,879

Kawka, A., \& Vennes, S. 2006, ApJ, 643, 402

Kawka, A., Vennes, S., \& Thorstensen, J. R. 2004, AJ, 127, 1702

Kawka, A., Vennes, S., Schmidt, G. D., Wickramasinghe, D. T., \& Koch, R. 2007, ApJ, 654, 499

Kemic, S. B. 1975, Ap\&SS, 36, 459

Kilic, M., von Hippel, T., Leggett, S. K., \& Winget, D. E. 2006, ApJ, 646, 474

Koester, D. 2009, A\&A, 498, 517

Koester, D., \& Wilken, D. 2006, A\&A, 453, 1051

Koester, D., Voss, B., Napiwotzki, R., et al. 2009, A\&A, 505, 441

Kowalski, P. M., \& Saumon, D. 2006, ApJ, 651, L137

Kupka, F., Ryabchikova T. A., Piskunov N. E., Stempels H. C., \& Weiss, W. W. 2000, Baltic Astron., 9, 590

Kurucz, R. L., \& Avrett, E. H. 1981, SAO Spec. Rep., 391 
A\&A 532, A7 (2011)

Luyten, W. J. 1977, White Dwarfs II (Minneapolis: Univ. Minnesota Press) Luyten, W. J. 1979, A catalogue of stars with proper motions exceeding 0.'5 annually (Minneapolis: Univ. Minnesota Press)

Luyten, W. J. 1980, NLTT Catalogue, Volume III, 0 to $-30^{\circ}$ (Minneapolis: Univ. Minnesota Press)

Martin, B., \& Wickramasinghe, D. T. 1981, MNRAS, 196, 23

Martin, N. J., Sandars, P. G. H., \& Woodgate, G. K. 1968, Roy. Soc. London Proc. Ser. A, 305, 139

Neale, L., \& Tennyson, J. 1995, ApJ, 454, L169

Nordhaus, J., Wellons, S., Spiegel, D. S., Metzger, B. D., \& Blackman, E. G. 2011, Proc. National Academy of Science, 108, 3135

Paquette, C., Pelletier, C., Fontaine, G., \& Michaud, G. 1986, ApJS, 61, 197

Patat, F., Moehler, S., O’Brien, K., et al. 2011, A\&A, 527, A91

Platais, I., Girard, T. M., Kozhurina-Platais, V., et al. 1998, AJ, 116, 2556
Pokorny, R. S., Jones, H. R. A., Hambly, N. C., \& Pinfield, D. J. 2004, A\&A, 421, 763

Potter, A. T., \& Tout, C. A. 2010, MNRAS, 402, 1072

Reid, I. N., Liebert, J., \& Schmidt, G. D. 2001, ApJ, 550, L61

Rohrmann, R. D., Althaus, L. G., \& Kepler, S. O. 2011, MNRAS, 411, 781

Salim, S., \& Gould, A. 2002, ApJ, 575, L83

Salim, S., \& Gould, A. 2003, ApJ, 582, 1011

Sion, E. M., Fritz, M. L., McMullin, J. P., \& Lallo, M. D. 1988, AJ, 96, 251

Sion, E. M., Kenyon, S. J., \& Aannestad, P. A. 1990, ApJS, 72, 707

Skrutskie, M. F., Cutri, R. M., Stiening, R., et al. 2006, AJ, 131, 1163

Tommaseo, G., Pfeil, T., Revalde, G., et al. 2003, Eur. Phys. J. D, 25, 113

Unno, W. 1956, PASJ, 8, 108

Wickramasinghe, D. T., \& Ferrario, L. 2005, MNRAS, 356, 1576

Zuckerman, B., Koester, D., Reid, I. N., \& Hünsch, M. 2003, ApJ, 596, 477 